\title{
ARIA-EAACI statement on severe allergic reactions to COVID-19 vaccines - an EAACI-ARIA Position Paper
}

\author{
Ludger Klimek ${ }^{1}$, Marek Jutel ${ }^{2}$, Cezmi Akdis ${ }^{3}$, Jean Bousquet ${ }^{4}$, Mubeccel Akdis ${ }^{5}$, María \\ José Torres $^{6}$, Ioana Agache ${ }^{7}$, Giorgio Walter Canonica ${ }^{8}$, Stefano R. Del Giacco ${ }^{9}$, Liam
} $\mathrm{O}^{\prime}$ Mahony ${ }^{10}$, Mohamed Shamji ${ }^{11}$, Ruby Pawankar ${ }^{12}$, Eva Untersmayr ${ }^{13}$, Johannes Ring ${ }^{14}$, Anna Bedbrook ${ }^{15}$, Margitta Worm ${ }^{16}$, and Torsten Zuberbier ${ }^{17}$

${ }^{1}$ Zentrum für Rhinologie und Allergologie

${ }^{2}$ Wrocław Medical University

${ }^{3}$ University of Zurich

${ }^{4}$ Université Versailles, St-Quentin-en-Yvelines

${ }^{5}$ University of Zürich

${ }^{6}$ Universidad de Málaga Facultad de Medicina

${ }^{7}$ Transylvania University of Brasov

${ }^{8}$ Humanitas Clinical and Research Center

${ }^{9}$ University of Cagliari Faculty of Medicine

${ }^{10}$ University College Cork National University of Ireland

${ }^{11}$ Imperial College London

${ }^{12}$ Nippon Medical School

${ }^{13}$ Center of Physiology, Pathophysiology and Immunology

${ }^{14}$ Technische Universitat Munchen

${ }^{15}$ Hôpital Arnaud de Villeneuve

${ }^{16}$ Charité - Universitätsmedizin Berlin

${ }^{17}$ Charité Universitätsmedizin Berlin

December 28, 2020

\begin{abstract}
Coronavirus disease 2019 (COVID-19) vaccine BNT162b2 received approval and within the first few days of public vaccination several severe anaphylaxis cases occurred. An investigation is taking place to understand the cases and their triggers. The vaccine will be administered to a large number of individuals worldwide and concerns raised for severe adverse events might occur. With the current information, the European Academy of Allergy and Clinical Immunology (EAACI) states its position for the following preliminary recommendations that are to be revised as soon as more data emerges. To minimize the risk of severe allergic reactions in vaccinated individuals, it is urgently required to understand the specific nature of the reported severe allergic reactions, including the background medical history of the individuals affected and the mechanisms involved. To achieve this goal all clinical and laboratory information should be collected and reported. Mild and moderate allergic patients should not be excluded from the vaccine as the exclusion of all these patients from vaccination may have a significant impact on reaching the goal of population immunity. Health care practitioners vaccinating against COVID-19 are required to be sufficiently prepared to recognise and treat anaphylaxis properly with the ability to administer adrenaline. A mandatory observation period after vaccine administration of at least 15 minutes for all individuals should be followed. The current guidelines, which exclude patients with severe allergies from vaccination with BNT162b2, should be re-evaluated after more information and experience
\end{abstract}


with the new vaccine develops.

\section{Hosted file}

EAACI PP-Severe allergic reactions to COVID-19 vaccines_22 122020 with abstract.pdf available at https://authorea.com/users/327586/articles/500993-aria-eaaci-statement-on-severeallergic-reactions-to-covid-19-vaccines-an-eaaci-aria-position-paper 